Brit. J. industr. Med., 1959, 16, 324.

\title{
THE ORIGIN OF THE TERM ANTHRACOSIS
}

\section{INTRODUCED BY}

\author{
A. MEIKLEJOHN \\ From the Department of Industrial Health, University of Glasgow
}

(RECEIVED FOR PUBLICATION DECEMBER 12, 1958)

\begin{abstract}
Dust diseases of the lungs are classified generically as the pneumoconioses. There are several varieties, of which anthracosis was the first to be named specifically. The original article, in which the name anthracosis was coined, is reprinted prefaced by a biographical note on its author.
\end{abstract}

"When I use a word" said Humpty Dumpty in a rather scornful tone, "it means just what I choose it to mean, neither more nor less". This quotation from "Alice in Wonderland" is not out of place here for it represents an attitude not infrequent in scientific writings. On occasion it is salutary to discover the precise circumstances and facts which led to the introduction of a new term.

In a previous article on the "History of Lung Diseases of Coal-miners in Great Britain" (Meiklejohn, 1951) I recounted the early clinical and pathological observations on black infiltration of the lungs and traced the development of the nomenclature from melanosis to spurious melanosis and finally to anthracosis.

The term "anthracosis", meaning charcoal lung, not black or coal lung, was introduced in 1838 by Dr. Thomas Stratton following attendance at the post-mortem examination on a coal-miner. It occurred to me that the original report would be of wide interest and worthy of reprinting especially as it is my intention later to publish a paper on Zenker's neologism, pneumonokoniosis.

Thomas Stratton was born at Perth, Scotland, in 1816. He lived there until he was 8 years old. The next five years were spent at Dunkeld on the border of the Highlands. From 1829 to 1833 he was in Northumberland and though there is no information on the point, he was probably engaged during this period as an apprentice apothecary. Thereafter he returned to Scotland where he studied medicine at Marischal College, Aberdeen, from 1834 until 1836. This was followed by a year at Edinburgh University. On April 18, 1837, he was admitted as a Licentiate of the Royal College of Surgeons of Edinburgh and on August 1, 1837, he was granted the degree of M.D., Edinburgh. His inaugural thesis was entitled "On Chronic Rheumatism". Then on December 19,
1837, he received his warrant as assistant-surgeon in the Royal Navy, where he spent his whole career. He was promoted Deputy Inspector General of Hospitals and Fleets in 1867. He died on March 16, 1886.

Throughout his life he wrote extensively on a wide variety of medical subjects. However, in our present purpose, it is his avocation which is of greatest interest. He himself records (Stratton, 1870) that in 1831 when he was aged 15 years he "procured a Gaelic Grammar and a Gaelic Dictionary, and some other works, and without any help, in the course of two or three months taught himself the language". This marked the beginning of his interest in languages with particular reference to comparative philology. He published essays - the first in 1840 at the age of 24 years - on the affinity of the Latin, Greek, and Hebrew languages to the Gaelic or Celtic of Scotland (1872). Thomas Stratton was truly a lad o' pairts.

\section{Case of Anthracosis or Black Infiltration of the Whole Lungs \\ BY}

THOMAS STRATTON, M.D.

"The interesting appearance, of which the following case is an instance, was first described by the late Dr. J. C. Gregory in 1831, and since that time several cases have been recorded.

On examining the bodies of elderly persons, we find the lungs always of a dark colour. Sometimes this colour is much deeper in various parts of the lung, which then are as black as charcoal; at other times the whole lungs are uniformly of this charcoal colour.

The first appearance is considered healthy; the second receives the name of melanosis; and the third is what has been called the black lung of coalminers, and may more shortly be defined anthracosis ( $a \nu \theta \xi \dot{a} \xi$, charcoal). 
On September 29th, 1837, I was invited by Dr. Crawford to witness the inspection of the body of a patient to Dr. Leitch. The history of this patient is as follows.

George Harrison had worked in a coal-mine for 50 years; at his death he was 70; for the last four years he had been an inmate of Tynemouth Workhouse; he had not worked in a coal-mine for some time previously. For some years before his decease, he had been affected with symptoms of chronic Bronchitis; the dyspnoea was not considerable; his expectoration was small in quantity, and never of a black colour. There was mucous rattle more on the left than the right side of the chest. On percussion, the sound was natural. Latterly he complained much of pain in the right hypochondrium, and had dropsical symptoms in the abdomen and inferior extremities.

Inspection.-Thorax.-On the left side the pleura pulmonalis and pleura costalis were connected by extensive and firm adhesions. Both lungs uniformly presented a perfectly black appearance externally and when cut into. A portion of them rubbed on the hand left a black stain, which was with some difficulty washed off, and a bit put into water gave it the colour of china-ink. Throughout both lungs were seen and felt hard masses of black matter from the size of a bean downwards. These masses were more numerous in the central parts of either lung, and also were in greater abundance in the central part of the left than of the right lung. In the left lung were several chalky bodies, encased in this black substance. No black substance was found in the bronchial glands. Nor was black matter seen in any other part of the body which was examined.

The heart was natural.

Abdomen.-A considerable quantity of fluid was found within the cavity of the peritoneum. The liver and spleen were much diminished in size. The kidneys were healthy.

Harrison's lung I compared with that of another subject, formerly a man-of-war's man, aged 72 . This lung was very much lighter in its colour than Harrison's and did not leave a stain when rubbed on the hand.

It is proper to mention that the coal-miners in this district do not use a lamp attached to the forehead as they do in the west of Scotland.

On the left side there was a greater number of black bodies, more pleural adhesion, and more bronchitis than on the right side. It is interesting to observe that these three conditions existed together.

If we suppose the matter of the anthracosis to have been inhaled by the man whilst at work in the mine, and that during the five or six years that he discontinued this kind of work, the lungs were endeavouring slowly to relieve themselves of the foreign matter, then the obstacle to free action of the left lung from pleural adhesion, and the smaller quantity of air which could be admitted along the left bronchial tubes thickened by inflammation, and partially obstructed by mucus, will explain how the anthracosis had become less in the right lung, when these states existed in a much less degree.

If the difference in the quantity of anthracotic matter in the two lungs is not to be explained in this way, then, either, more inhaled black matter has been originally received by the left lung or the function of the elimination of carbon has been less imperfectly performed in the right than in the left lung. With respect to black expectoration in cases of anthracosis, I may refer to the instructive paper by Dr. William Thomson, in the London Medico-Chirurgical Transactions for 1836, and of which a short account was given in the last Number of this Journal (January 1838 , p. 260).

Dr. Cumin's patient (Edinb. med. surg. J., vol. xlii, p. 324) appears to have been affected with phthisis pulmonalis, complicated with anthracosis, as phthisis sometimes is with melanosis. Dr. Laurie's and Dr. Buchanan's cases (ib. pp. 328, 329) prove the existence of anthracosis without the slightest pectoral affection. Dr. Buchanan's case of Lyall seems to have been anthracosis along with peritoneal melanosis.

Melanosis of the lung appears to differ from anthracosis in the following particulars. Melanosis exists in isolated portions, while anthracosis is general over the lung. Of melanosis, the local effects are dyspnoea, cough, which is often dry, but sometimes attended with mucous expectoration, and mixed with some puriform sputa, while anthracosis may exist without any chest symptoms whatever. Of melanosis, the most constant constitutional symptoms are the gradual diminution of the vital powers, and diminished nutrition followed by hydropic affections, whereas anthracosis often is unexpectedly found to be present when death has occurred from other causes. In melanosis, black deposits are generally found in other parts of the body besides the lungs, whilst anthracosis is met with in these alone; and lastly, the matter of melanosis loses its black colour when treated with chlorine (Dr. Henry), whereas the matter of anthracosis retains its colour when so treated (Professor Christison)."

\section{REFERENCES}

Meiklejohn, A. (1951). Brit. J. industr. Med., 8, 127. Stratton, T. (1838). Edinb. med. surg. J. 49, 490.

- (1870). The Celtic Origin of a Great Part of the Greek and Latin Languages, 2nd ed. Edinburgh: Maclachlan and Stewart. Celtic, 3rd ed. Edinburgh: Maclachlan and Stewart. 